\title{
Frequency-Domain Channel Estimation for SC-FDE in HF Channel
}

\author{
Qingyun Zhu, Xu He, Shaoqian Li \\ National key Lab of Communication, University of Electronic Science and Technology of \\ China ,Chengdu, 610054, P. R. China \\ zhuqingyun1@qq.com;hexu@uestc.edu.cn
}

\begin{abstract}
Keyword: Frequency domain channel estimation. Sliding pilot block. Set small energy paths to zero.
\end{abstract}

\begin{abstract}
HF channel is a common multipath propagation resulting in frequency selective fading, SC-FDE can better resist frequency selective fading, but commonly used frequency domain equalization algorithm needs to get channel estimates. In this paper, the research group is applicable in the HF channel frequency domain channel estimation. The algorithm after the least-squares (LS) channel estimation algorithm, used methods that are the sliding pilot blocks and set small energy paths to zero to eliminate Gaussian noise and then used the interpolation algorithm to reduce the number of pilot in the frame structure to improve the transmission efficiency and track the fading of HF channel. Through the simulation prove that, the algorithm has good use to estimate the channel estimates also improve the spectrum utilization efficiency.
\end{abstract}

\section{Introduction}

Sky wave transmission of the HF communication is influenced by the ionosphere seriously which lend to more serious multipath effect ${ }^{[1]}$. Multipath effect will lead to frequency selective fading, broaden the waveform of received signal and produce inter symbol interference (ISI) which limits the transmission performance and transmission efficiency of digital communication. The existing multicarrier orthogonal frequency division multiplexing (OFDM) and single carrier time-domain equalization technology (SC-TDE) in combating multipath fading channel has good performance, but OFDM suffers from peak-to-average power ratio (PAPR) are relatively high and also have the disadvantages of sensitive to carrier frequency offsets, and the computation complexity of TDE is considerable high to handle severe ISI due to high speed data transmission in SC-TDE system. Single carrier frequency domain equalization $(\mathrm{SC}-\mathrm{FDE})^{[2]}$ technique with single carrier transmission overcomes the disadvantage of PAPR are relatively higher in OFDM and the frequency domain equalization overcomes the disadvantage of time domain equalization with large amount of computation.

HF channel is composed of many propagation paths with different time delays and has a narrow bandwidth lead to high rate transmission is so difficult and characterized by a severely frequency-selective channel. In this paper, used sliding pilot block and set small energy path to zero to obtain accurate value of channel estimation and then used interpolation algorithm to track the fading of HF channel. The proposed channel estimation algorithm could improve the transmission performance of system.

\section{Conventional Sc-Fde System Description}

SC-FDE baseband signal processing block diagram is shown in Figure 1. In the transmitter, after the process of modulation, the data blocks which length is $N$ then insert Unique Word (UW) that length is $N_{g}$ and receiver is known. The $N_{g}$ must be greater than the value of multipath time delay symbol length is result by the biggest path delay of channel. For example, generally in the HF channel considered the biggest path delay of channel is $5 \mathrm{~ms}$ to fully estimate the channel impulse response (CIR). After the synchronization of receiver, extraction of each block UW sequence to estimate channel frequency response of the current time, then via the FFT block, then transmit the data blocks to frequency domain to process frequency domain equalization. After that put the signal 
to process IFFT get the time domain signal. At last, remove the UW sequences and demodulate the time domain signal are in order to restore bits. Different from the structure of time domain equalization, the coefficients of frequency domain equalization is multiple single filter is same to the frequency domain form of the time domain filter coefficient.

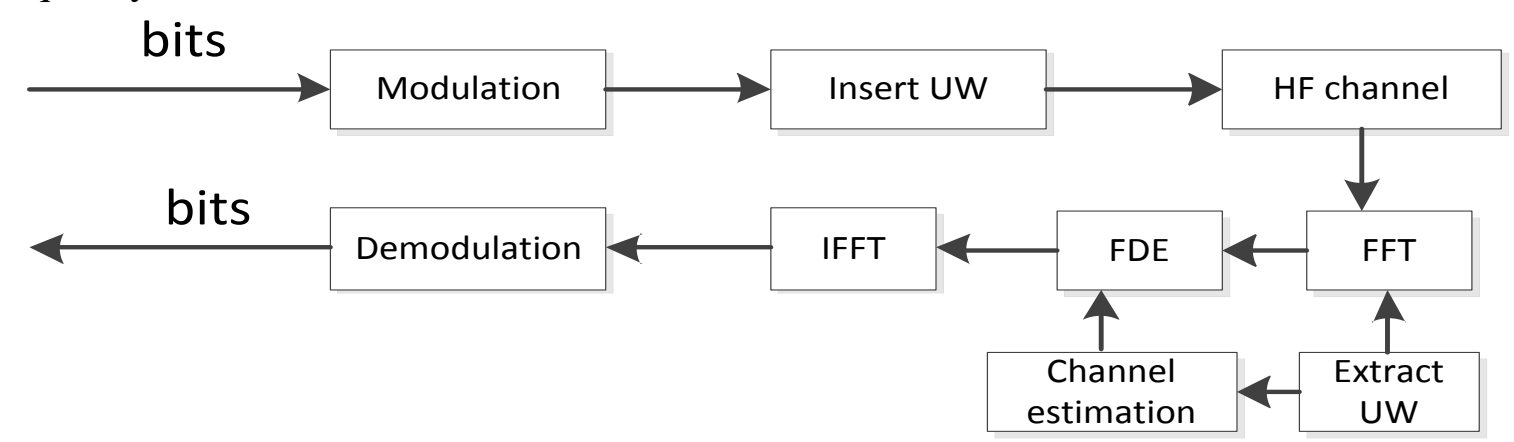

Fig.1. The signal processing flow of SC-FDE

In the SC - FDE system, UW sequence can be used as cyclic prefix (CP), which can used to pilot sequences to do channel estimation too. As the UW sequence been the $\mathrm{CP}$, the length of data must be greater than the length of the response of the channel in order to eliminate ISI. As the UW sequence been the pilot sequences, for UW sequences with good correlation and cyclical, smooth frequency response. According to the IEEE802.11a standard commonly use UW sequence such as: $\mathrm{Chu}^{[3]}$, Frank-Zadaff, PN sequences, etc. In this paper, use the Chu sequence as UW sequence. The conventional frame structure is shown in Figure 2.

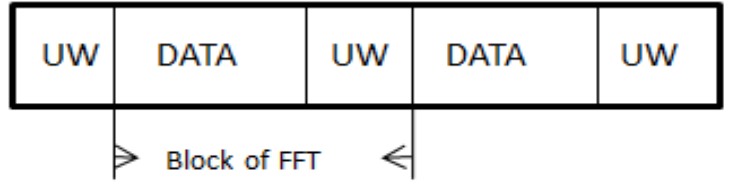

Continuous transmission mode

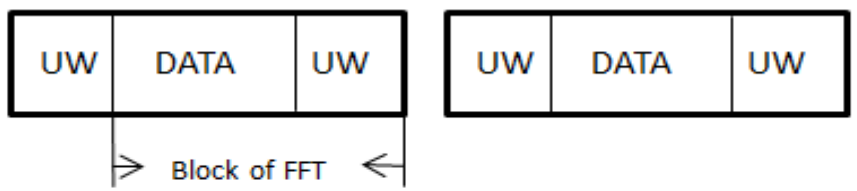

Abrupt transmission mode

Fig.2. Conventional structure of frame

The time domain impulse response (CIR) of multipath fading channel can be represented as:

$$
h(n)=\sum_{l=0}^{L-1} h_{l} \exp \left(\frac{j 2 \pi f_{D l} T n}{N}\right) \delta\left(\tau-\tau_{l}\right)
$$

where $L$ is the total number of paths, $f_{D l}$ is Doppler frequency shift of $l_{t h}, \tau_{l}$ is the time delay of $l_{t h}, T$ is the cycle of a transmission block, $T=N T_{s}, T_{s}$ as the sampling period, $n$ as the sampling time.

The transmit data is additive white Gaussian noise (AWGN) in the channel. In the receiver, the received signal can be represented as:

$$
\mathrm{y}(\mathrm{n})=x(n) * h(n)+v(n)
$$

where $*$ in (2) is convolution symbol, $x(n)$ represented as transmit signal, $h(n)$ represented as the channel impulse response, $v(n)$ is AWGN channel of mean to 0 , variance for sigma is $\sigma^{2}$. In the ideal state, Because of the $\mathrm{CP}$, the linear convolution could convert to cyclic convolution, namely:

$$
\mathrm{y}(\mathrm{n})=x(n) \mathrm{X} h(n)+v(n)
$$

In type (3) on both sides use Fourier transform which can get the expressions of the frequency domain can be represented as:

$$
Y(k)=X(k) H(k)+V(k)
$$


where $H(k)$ is discrete Fourier transform of $h(n)$, that is channel frequency response. Discrete Fourier transform of $x(n), y(n), v(n)$ is $X(k) 、 Y(k) 、 V(k)$.

\section{Channel Estimation}

\section{The LS channel estimation algorithm.}

Line frequency domain equalization algorithm used MMSE, ZF commonly, which need the value $H_{L}$ to offset ISI. In order to get the $H_{L}$, must be on channel estimation. At present, channel estimation algorithm used least square (LS) channel estimation algorithm is proposed commonly which compare to minimum mean square error (LMMSE) channel estimation algorithm that has advantages of small amount of calculation and easy to realize. In this paper, the channel estimation algorithm use LS channel estimation.

LS channel estimation is according to the least squares criterion to get the channel estimation algorithm. In (2-4) can be represented by matrix as: $Y=X H+V$. According to the least squares criterion, defining the cost function as:

$$
J_{L S}=(Y-X H)^{H}(Y-X H)
$$

where $X$ is pilot sequences that is known by reception, $X=\operatorname{diag}\left[X(0), X(1), \ldots X\left(N_{g}-1\right)\right]$. $Y$ is pilot sequences signal via the channel to receive the column value, $Y=\operatorname{diag}\left[Y(0), Y(1), \ldots Y\left(N_{g}-\right.\right.$ $1)]^{T} . H$ is the pilot position corresponding the channel frequency response column value, $H=$ $\operatorname{diag}\left[H(0), H(1), \ldots H\left(N_{g}-1\right)\right]^{T} . N_{g}$ is the length of the pilot sequences. In order to get best channel estimate of $H$, so the $J_{L S}$ about $H$ the first-order and second-order derivatives:

$$
\begin{aligned}
& \frac{\partial}{\partial H} J_{L S}=-2 X^{H}(Y-X H) \\
& \frac{\partial}{\partial H}\left(\frac{\partial}{\partial H} J_{L S}\right)^{H}=2 X^{H} X \geq 0
\end{aligned}
$$

Could be concluded that the second derivative is bigger than zero, there exist a minimum value. Order first derivative is zero, you can get:

$$
\widehat{H}_{L S}=\left(X^{H} X\right)^{-1} X^{H} Y=\frac{Y}{X}
$$

The LS channel estimation all operations in frequency domain, because of its simple structure, so it has been widely used. But as can be seen in (8), The LS channel estimation without considering the noise cancellation. So the value of LS is influenced by noise. The relationship between real channel parameters $H$ and $\widehat{H}_{L S}$ is:

$$
\widehat{H}_{L S}=X^{-1} Y=X^{-1}(X H+V)=H+X^{-1} V=H+n^{\prime}
$$

Used the value of channel estimation by LS channel estimation is be as an initial value of channel estimation.

\section{Sliding Pilot Block Channel Estimation.}

The influence of Gaussian white noise on the channel coefficients mainly for two aspects: the first is that the channel estimation value of main paths is fluctuated caused by Gaussian noise. The second is that there will appear many small energy paths in coefficients of channel estimation that is not existence in real channel. In actual wireless communication channel has "sparse multipath" features (The energy of channel impulse response mainly focus on a few sampling points). The small energy paths which is existence in real channel will has a great influence on the performance.

First of all, in order to eliminate noise on the main paths, an improved frame structure as shown in Figure 3. This frame structure compare with conventional SC-FDE frame structure the difference is each pilot block composed by two UW sequences, based on the improved frame structure proposed a sliding pilot block channel estimation which use adjacent UW blocks. 


\begin{tabular}{|l|l|l|l|l|l|l|l|}
\hline UW & UW & DATA & UW & UW & DATA & UW & UW \\
\hline
\end{tabular}

Improved continuous transmission mode

\begin{tabular}{|l|l|l|l|l|l|l|l|l|l|l|}
\hline UW & UW & DATA & UW & UW & UW & UW & DATA & UW & UW \\
\hline
\end{tabular}

Improved abrupt transmission mode

Fig. 3. Improved frame structure

This algorithm basic idea is processed $K$ times LS channel estimation for same data of block and then to seek an average of the $K$ times LS channel estimation value.

Firstly, used $i$ and $i+1$ block of UW sequences which length is $N_{g}$ to form a pilot block which length is $2 N_{g}$, such as: $\mathrm{y}=\left[y_{i}, y_{i+1}\right]=\left[\hat{y}_{i}(0), \ldots, \hat{y}_{i}\left(N_{g}-1\right), \hat{y}_{i+1}(0), \ldots, \hat{y}_{i+1}\left(N_{g}-1\right)\right]$ and then used any continuous sequences $q_{a}=\left[q_{a}(0), \ldots, q_{a}\left(N_{g}-1\right)\right]$ which length is $N_{g}$ in y to estimate the coefficients of channel. The most times of channel estimation is $N_{g}$ if use the improved frame structure. Such as $q_{a}$, the $a$ of $q_{a}$ is the start position in the sequence $\mathrm{y}$, the $q_{a}$ is getting UW sequences that length is $N_{g}$ by turn in y which length is $2 N_{g}$. Each $q_{a}$ sequence could get a set of channel estimation value, at last, can obtain an mean value of channel estimation by average all the value of channel estimation that could reduce the effect of Gaussian noise. Such as to obtain continuous UW sequences which number is $K$ :

$\widehat{H}(i)=\frac{1}{K} \sum_{a=0}^{K-1} \widehat{H}_{a}(i)$

$=\frac{1}{K} \sum_{a=0}^{K-1}\left(H_{a}(i)+n^{\prime}{ }_{a}(i)\right)$

$=\frac{1}{K} \sum_{a=0}^{K-1} H_{a}(i)+\frac{1}{K} \sum_{a=0}^{K-1} n^{\prime}{ }_{a}(i)$

Show in (10), When $K$ tends to infinity, the item of noise tends to 0.namely:

$\frac{1}{K} \sum_{a=0}^{K-1} n_{a}^{\prime}(i)=0$

So that could achieve the purpose of noise cancellation. But the actual can't take the value of $K$ in infinity, should consider the performance of system and the amount of calculation to decide the size of $K$.

Set small Energy Paths to Zero.

Sliding pilot block channel estimation is to eliminate the noise of mainly paths and can't take the value of $K$ in infinity, so should find other method to eliminate the residual noise. Conventional LS channel estimation will produce many small energy paths caused by Gaussian noise, HF channel is "sparse multipath" could use set small energy paths to zero to eliminate the residual noise caused by small energy paths, a large amount of measured data statistics in ITU- $\mathrm{R} \mathrm{F}^{[1]}$. agreement represent that the number of multipath is $2 \sim 4$ in HF channel commonly, among them the number of multipath is 3 have the highest probability then is the number of multipath is 2 and 4 . Reserved the number of biggest channel estimation coefficients is $n$. The number of $n$ should be in $5 \sim 10$ in HF channel. Then set other paths to be 0 .In this paper set $n=6$.

Specific means is when get the value of channel estimation $\widehat{H}=\left[\widehat{H}(0), \ldots, \widehat{H}\left(N_{g}-1\right)\right]$ by LS. Transformed $\widehat{H}$ to time domain $\hat{h}$ by IDFT operation to obtain $\widehat{h}=\left[\hat{h}(0), \ldots, \hat{h}\left(N_{g}-1\right)\right]$, compare the size of $\hat{h}(i),\left(0 \leq i \leq N_{g}-1\right)$ to reserve the biggest $\hat{h}(i)$ which number is $n$ and set the other $\hat{h}(i)$ that number is $N_{g}-n$ to be 0 , then can get $\hat{h}_{z}$ which is eliminated noise of small energy paths. At last, transformed $\hat{h}_{z}$ which adopt set small energy paths to zero to $\widehat{H}_{z}$ by 
DFT operation. Figure 4 is a frequency spectrum line is used set small energy paths to zero to eliminate noise which is more close to the line of ideal Gaussian noise cancellation compare to the line of add Gaussian noise.

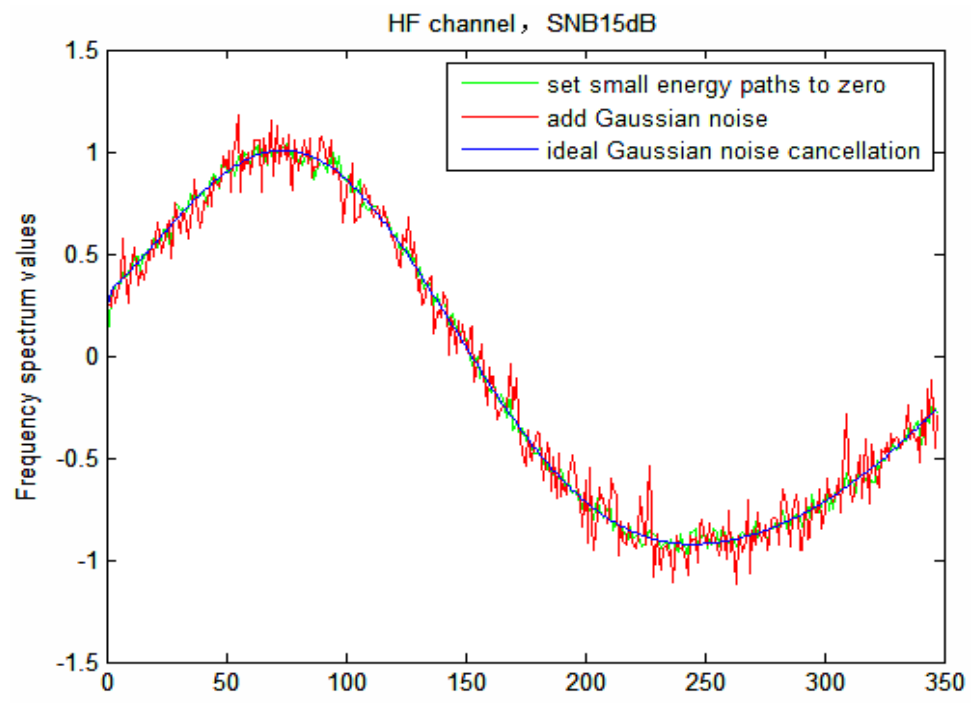

Fig.4. Set small energy paths to zero to eliminate noise

Adopted sliding pilot block channel estimation and set small energy paths to zero can get an accurate value of channel estimation by eliminating Gaussian noise.

Pilot Block Interpolation Channel Estimation Algorithm.

Conventional LS channel estimation assumed channel to be a static channel within a block of data, that is in the duration of a block of data assumes that the channel impulse response remains the same. Due to the HF channel under influence of multipath effect, Doppler frequency shift and the Doppler spread lead to fad frequently, in order to obtain accurate value of channel estimation should track the fading of HF channel. In conventional SC-FDE system used many UW sequences to improve the performance of the system but reduce the transmission efficiency of the system. Based on the situation, proposed a method of reduce pilot sequences and used the interpolation algorithm to obtain all data blocks corresponding channel estimates value. Figure 3 is based on the interpolation algorithm design of frame structure.

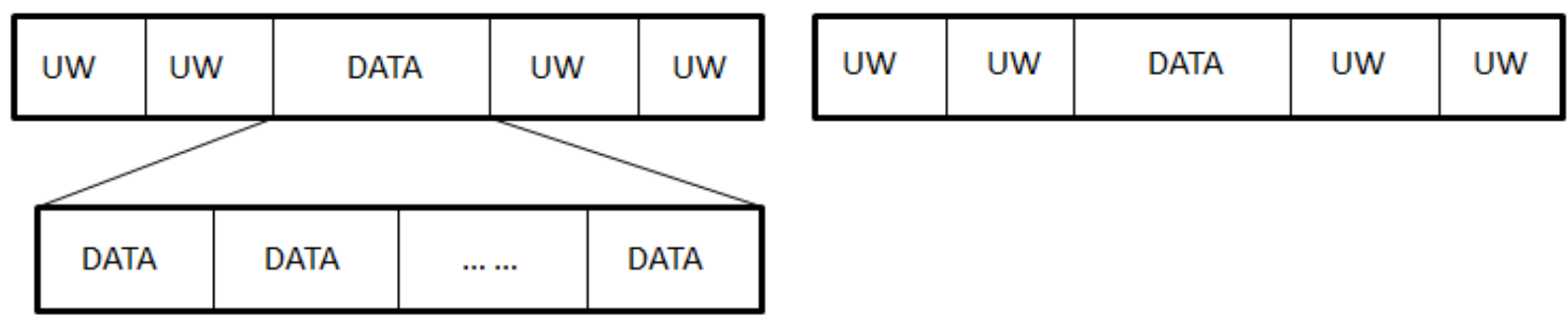

Improved abrupt transmission mode with interpolation

Fig.5. The improved frame structure of abrupt transmission mode

In the conventional system is a pilot sequences connect a block of data, but in the improved frame structure is a pilot sequences connect many blocks of data. For example, in figure 5 frame structure used fore and aft pilot blocks to get channel estimation $\widehat{H}_{a}, \widehat{H}_{b}$. Then the channel estimation of data $\widehat{H}_{1} \ldots \widehat{H}_{\mathrm{i}-1}(i$ is the number of data blocks) are obtained through the interpolation algorithm.

If channel time varying is not fast and decline is not serious could use line interpolation algorithm to get the value of channel estimation. But in HF channel, the fading is frequently, the low order interpolation algorithm to get the accurate channel estimation value of data is hardly, could use high order interpolation algorithm to do that. The commonly used high order 
interpolation algorithm such as: spline, cubic, etc. Using high order interpolation algorithm need more reference point (pilot sequences) to improve the estimation accuracy. So if the transmission mode is continuous transmission, the frame structure as shown in Figure 6.

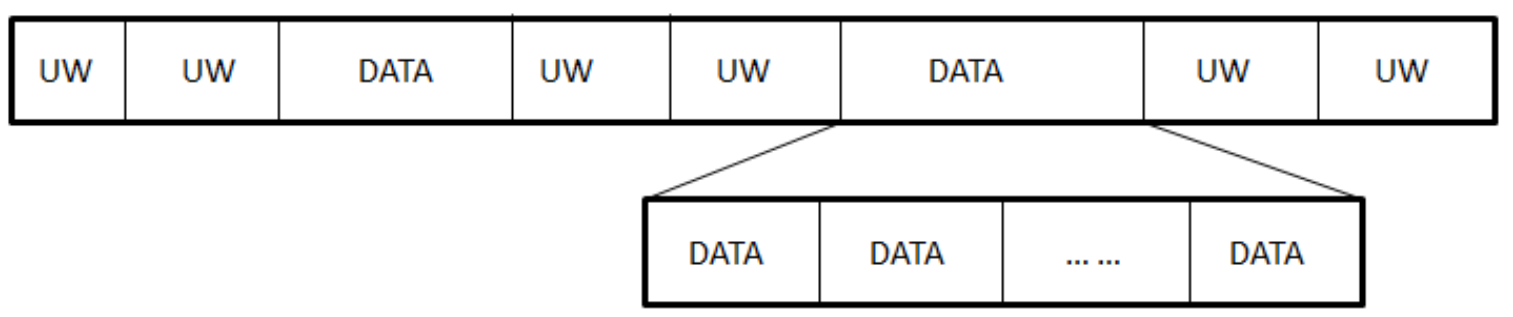

Improved continuous transmission mode with interpolation algorithm

Fig.6. The improved frame structure of continuous transmission mode

In Figure 4 can get 4 values of channel estimation through 4 blocks of UW sequences according to the LS channel estimation algorithm respectively. $\widehat{H}_{e s t i}=\left[\widehat{H}_{1}, \widehat{H}_{6}, \widehat{H}_{11}, \widehat{H}_{16}\right]$. Then used interpolation algorithm to get $\widehat{H}_{e s t i}$, inserted $N$ data between $\widehat{H}_{i}$ and $\widehat{H}_{i+1} . N$ is the number of blocks of data that is between UW sequences. In Figure $4 N$ is 4 . After that will get the value of channel estimation block of data $\widehat{H}=\left[\widehat{H}_{2}, \widehat{H}_{3}, \widehat{H}_{4}, \widehat{H}_{5}, \widehat{H}_{7}, \widehat{H}_{8}, \widehat{H}_{9}, \widehat{H}_{10}, \widehat{H}_{12}, \widehat{H}_{13}, \widehat{H}_{14}, \widehat{H}_{15}\right], \widehat{H}$ is the value of channel estimation about the 12 blocks of data respectively. Figure 7 is a frequency spectrum curve line is used interpolation to estimate channel which is more close to the line of a fading frequency domain spectrum in HF that can prove used spline interpolation could track the fading of HF channel.

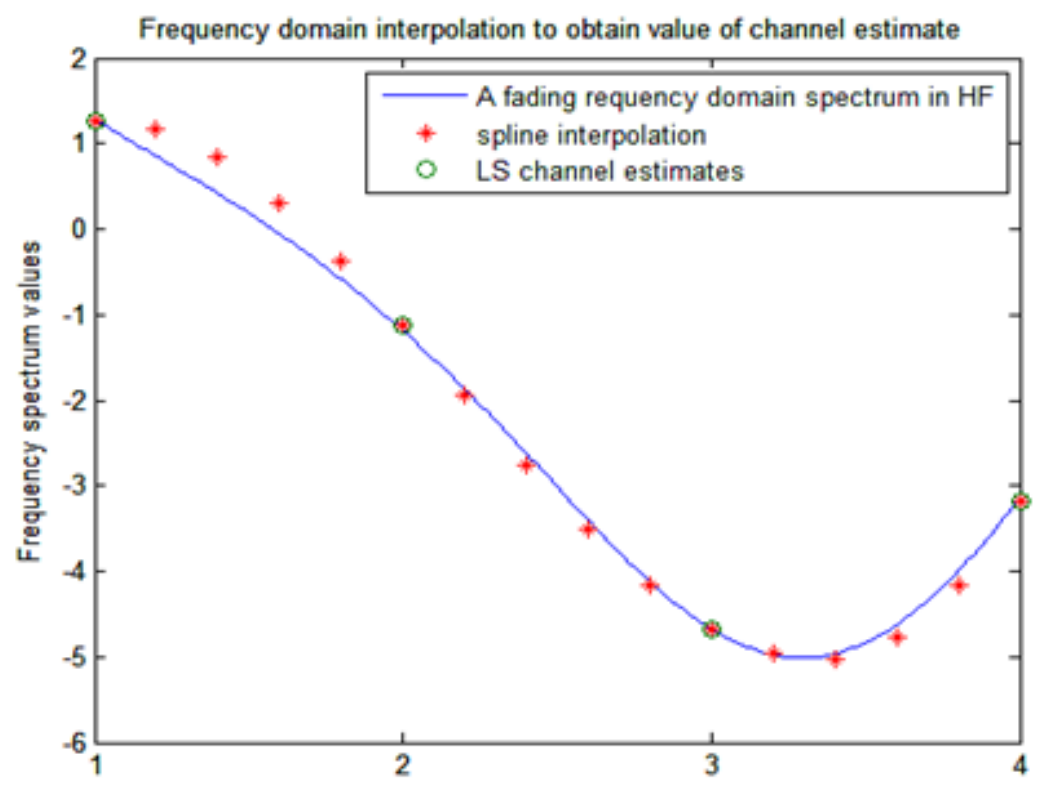

Fig.7. Interpolation algorithm tracking the fad of HF channel

The number and length of data blocks should be adjusted according to different channel, the number and length of data blocks may be appropriately increased when the situation of channel is better. When channel condition is bad should decrease the number and length of data blocks to make data transmission correctly. This method use fewer pilot sequences get the location of channel estimation, then use interpolation algorithm to get each channel estimation value of block data. Compared with conventional SC-FDE system, the interpolation algorithm saves many pilot blocks. The spectrum resource is very nervous in HF channel, use this method decrease number of pilot block to improve the transmission efficiency. 


\section{Simulation Results}

The bit error rate (BER) performance of improved SC-FDE is simulated with assumption that the synchronization is perfect and without coding. Used HF channel that bandwidth is $3 \mathrm{KHz}$, Doppler frequency spread is $1.5 \mathrm{~Hz}$, the multipath is 2 and the biggest time of delay is $2 \mathrm{~ms}$.used BPSK modulation. The monte carlo simulation times is 5000 .

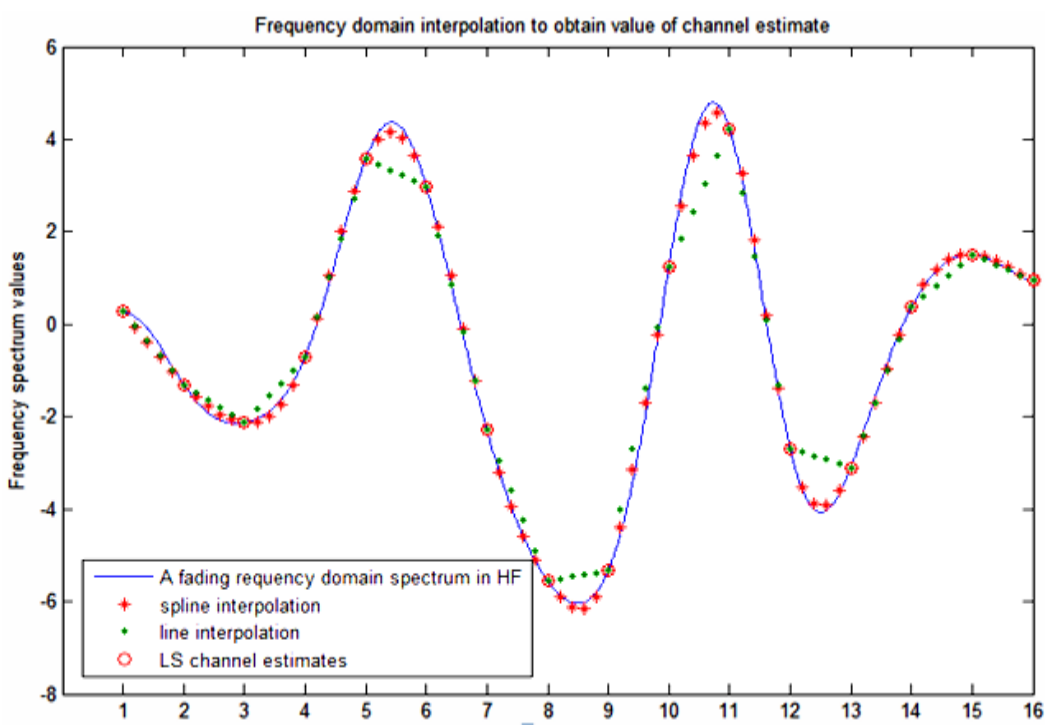

Fig.8. Compare different interpolation algorithm

As can be seen in Figure 8, consider LS value of channel estimation is perfect without consider Gaussian noise. The spline interpolation is better than line interpolation. The line used spline interpolation is more close to the real frequency fading curve line which can have better performance to track the fading of HF channel. The rest of the simulation use spline to interpolate.



Fig.9. Compare different channel estimation algorithm

As can be seen in Figure 9, the frame structure of channel estimation without interpolation is Figure 3, the length of FFT block which is not to use interpolation is 512, the length of UW is 32 . The frame structure of channel estimation with interpolation is Figure 5, the length of FFT block which is using interpolation are 128, then there are 4 DATA in the frame, in total the length of data is $128 * 4=512$. Used interpolation algorithm to reduce the length of FFT block can improve the performance of system and spectrum utilization efficiency. Both set small energy paths to zero and sliding pilot block channel estimation can improve accuracy of the estimated and have better 
performance compare with LS channel estimation. Used the denosie algorithm to get accurate value of channel estimation and then used interpolation to track the fading of channel can get better system performance compare to which without use interpolation have about $6 \mathrm{~dB}$ improvement in Fig.9.



Fig.10. Analysis channel estimation in continuous transmission mode

As can be seen in Figure 10, used frame structure is Figure 6, the number of DATA between UW sequences are 4 or 8 , the length of DATA are 256 and 128 respectively. Both of them used 4 LS channel estimation to estimate 12 or 24 blocks of data, this method reduced calculation of channel estimation compared with conventional SC-FDE. The Figure also proved small block of data and use eliminate the Gaussian noise have better performance of system. If used overlap FDE instead of conventional MMSE in interpolation channel estimation algorithm can improve transmission efficiency of system.

\section{Conclusion}

In this paper, we have proposed a Frequency-Domain channel estimation for SC-FDE in HF Channel that is used sliding pilot block channel estimation and set small energy paths to zero to eliminate Gaussian noise to get accurate value of channel estimation and then used the value to track the fading of HF channel by interpolation channel estimation algorithm. Used these methods can improve the performance of system. The interpolation channel estimation algorithm can use with OVERLAP-FDE can improve transmission efficiency of system to save spectrum resources of the HF channel. These methods can use in other fading channel too, according to different channels could choose different length of DATA and interpolation algorithm.

\section{Reference}

[1] ITU-R F.520 Use OF High Frequency Ionospheric Channel Simulators [S]. 1992.

[2] H Sari, G Karma, Jean Klaude. Frequency Domain equalization of mobile radio and terrestrial broadcast channels. IEEE Global Telecommunications Comference.Vol.1.pp 1-5.1994.

[3] David C. Chu. Poly phase Codes with Good Periodic Correlation Properties. IEEE Transactions on Information theory. Jul.1972.pp:531-532.

[4] Ashraf A. Tahat. MULTI-USER CHANNEL ESTIMATION IN A 4G OFDM SYSTEM. The 18th Annual IEEE International Symposium on Personal, Indoor and Mobile Radio Communications (PIMRC'07). 2007 IEEE. 
[5] David Falconer, Sirikiat Lek Ariyavisitakul, Anader Benyamin-Seeyar. Frequency Domain Equalization for Single-Carrier Broadband Wireless Systems. IEEE Comm. Vol.40.pp.58-66. April 2002.

[6] N. Benvenuto and S. Tomasin, "Iterative design and detection of a DFE in the frequency domain," IEEE Trans. Commun., vol. 53, no. 11, pp.1867-1875, Nov. 2005.

[7] Cai B, Zhao M J, Qiu P L. A novel channel estimation method for SC-FDE over fast fading channels[C].Communication Technology, 2006. ICCT'06. International Conference on. IEEE, 2006: 1-4. 